\title{
A Definition for Hesitant fuzzy Partitions
}

\author{
Laya Aliahmadipour, ${ }^{1}$ Vicenç Torra, ${ }^{2}$ Esfandiar Eslami, ${ }^{3}$ Mahdi Eftekhari ${ }^{4}$ \\ ${ }^{1}$ Department of Applied Mathematics, Faculty of Mathmatics and Computer, \\ Shahid Bahonar University of Kerman, Pajoohesh square, \\ Kerman, Iran \\ L.Aliahmadipour@math.uk.ac.ir \\ ${ }^{2}$ School of Informatics, Skövde University, \\ Högskolan i Skövde, \\ Högskolevägen, \\ Skövde, Sweden \\ vtorra@his.se \\ ${ }^{3}$ Department of Pure Mathematics, Faculty of Mathematics and Computer, \\ Shahid Bahonar University of Kerman, Pajoohesh square, \\ Kerman, Iran \\ Esfandiar.Eslami@uk.ac.ir \\ ${ }^{4}$ Department of Computer Engineering, Faculty of Engineering, \\ Shahid Bahonar University of Kerman, Pajoohesh square, \\ Kerman, Iran \\ M.Eftekhari@uk.ac.ir
}

Received 25 November 2015

Accepted 5 February 2016

\begin{abstract}
In this paper, we define hesitant fuzzy partitions (H-fuzzy partitions) to consider the results of standard fuzzy clustering family (e.g. fuzzy c-means and intuitionistic fuzzy c-means). We define a method to construct $\mathrm{H}$-fuzzy partitions from a set of fuzzy clusters obtained from several executions of fuzzy clustering algorithms with various initialization of their parameters. Our purpose is to consider some local optimal solutions to find a global optimal solution also letting the user to consider various reliable membership values and cluster centers to evaluate her/his problem using different cluster validity indices.
\end{abstract}

Keywords: Fuzzy partition, I-fuzzy partition, Hesitant fuzzy set, Hesitant fuzzy partition.

\section{Introduction}

Data clustering is the process of discovering natural groupings or clusters within multidimensional data based on some similarity or dissimilarity measure ${ }^{1}$. Clustering algorithms have been studied for decades. Many clustering algorithms have been developed until now, but none of them is proper for all purposes. Some clustering algorithms are suitable for dealing with data of certain types, and some are suitable for handling data with special distribution structures. Many real data have complex distribu- 
tions with noise and isolated points and, they are in high dimensional spaces. So there is a continuous demand for researching different kinds of clustering methods ${ }^{2}$. In order to obtain better clustering results in real-world applications, some researchers try their best to provide new efficient and effective clustering algorithms.

Most clustering algorithms are sensitive to the selection of initial parameters. For instance the clustering results of fuzzy c-means with various kernels and initial cluster centers selection methods are diverse. The choice of a clustering algorithm depends on the type of data available and the particular purpose $^{3}$. Thus, for a data set without any prior knowledge, we have difficulties on selecting the clustering algorithm, the kernel and the initial cluster centers. In order not to miss the proper clusters, we can consider the application of different clustering algorithms. We use hesitant fuzzy set to consider more than one fuzzy clustering result. In this study we consider more than one fuzzy clustering algorithm at the same time to avoid losing relevant information. To this end we apply fuzzy clustering algorithms using various initial parameters and executions and the results are modeled by a hesitant fuzzy partition (Hfuzzy partition).

The concept of Hesitant Fuzzy Sets (HFSs) has been introduced ${ }^{4,5}$ to model the uncertainty that often appears when it is necessary to establish the membership degree of an element and there are some possible values that make us to hesitate about which one would be the right one. Recently, many researchers have studied this concept who have proposed diverse extensions (dual hesitant fuzzy set ${ }^{6}$, generalized hesitant fuzzy set ${ }^{7}$ ), different types of operators to compute with this type of information, applications on clustering, decision-making, information fusion, etc ${ }^{8}, 9$.

In this study, we apply hesitant fuzzy sets in a clustering context, and use them to consider and aggregate some clustering results. We also define a new set of cluster centers and set of membership values that are used in various cluster validity indices in different situation. To this end the reminder of this paper is organized as follows. Section 2 reviews some related concepts. Section 3 presents a defini- tion of H-fuzzy partition and an example. Conclusions and future work are presented in section 4.

\section{Preliminaries}

In this section, we present some basic concepts related to fuzzy partitions, intuitionistic fuzzy partitions (I-fuzzy partition) and hesitant fuzzy sets to define H-fuzzy partitions in the next section.

\subsection{Fuzzy Partitions}

Most fuzzy clustering methods and in particular fuzzy c-means and related algorithms, construct a fuzzy partition of a given dataset that follows the next definition.

Definition 1. ${ }^{10}$ Let $X$ be a reference set. Then, a set of membership functions $M=\left\{\mu_{1}, \cdots, \mu_{n}\right\}$ on $X$ is a fuzzy partition of $X$ if for all $x \in X$ it holds $\sum_{i=1}^{n} \mu_{i}(x)=1$.

Note that not all fuzzy clustering algorithms lead to membership functions of this form e.g., possibilistic clustering ${ }^{11,12}$ does not require that memberships add to one. Among the various fuzzy clustering techniques, the most widely used ones include fuzzy c-means $(\mathrm{FCM})^{13}$ and their variants.

\subsubsection{Fuzzy c-means}

Fuzzy C-means (FCM) was introduced by Bezdek et al. in ${ }^{13}$. It is an unsupervised algorithm for fuzzy clustering that attracted scientists attention. The algorithm works to minimize an objective function that is defined as:

$$
J_{F C M}(U, V, X)=\sum_{i=1}^{N} \sum_{j=1}^{C} \mu_{i j}^{m} d^{2}\left(x_{i}, v_{j}\right),
$$

subject to the constraints $\mu_{i j} \in[0,1]$ and $\sum_{j=1}^{C} \mu_{i j}=$ 1 for all $x_{i} \in X$. In the above equation, $X=$ $\left\{x_{1}, \cdots, x_{i}, \cdots, x_{N}\right\}$ is a matrix of input objects, $N$ is the number of objects and $C$ is the number of clusters. $V=\left\{v_{1}, \cdots, v_{j} \cdots, v_{C}\right\}$ is the set of centers of clusters and $U_{N \times C}=\left\{\mu_{i j}\right\}$ is a fuzzy membership function matrix. The element $\mu_{i j}$ represents the degree of belongingness of the ith object to the jth 
cluster. $m$ is the fuzzy index that governs the influence of membership grades ( $m$ is any real number greater than 1, in general, $m$ is set to 2 and $d^{2}\left(x_{i}, v_{j}\right)$ is squared norm distance, which is used for measuring the dissimilarity between $x_{i}$ and $v_{j}$ :

$$
d^{2}\left(x_{i}, v_{j}\right)=\left\|x_{i}-v_{j}\right\|^{2} .
$$

The FCM is usually solved as follows. The algorithm begins by initializing the centers vectors randomly. Then an iterative process is applied including two steps. First $\mu_{i j}$ are computed using the following equation:

$$
\mu_{i j}=\frac{1}{\sum_{k=1}^{C}\left(\frac{d^{2}\left(x_{i}, v_{j}\right)}{d^{2}\left(x_{i}, v_{k}\right)}\right)^{\frac{2}{m-1}}} \quad \forall i=1, \cdots, N .
$$

Next, the centers are updated using

$$
v_{j}=\frac{\sum_{i=1}^{N}\left(\mu_{i j}\right)^{m} x_{i}}{\sum_{i=1}^{N}\left(\mu_{i j}\right)^{m}} \quad j=1, \cdots, C, \quad 1<m<N .
$$

Then the iterative process is repeated with new memberships and centers until $\left|v_{j}^{t+1}-v_{j}^{t}\right|<\varepsilon$ and $\left|\mu_{i j}^{t+1}-\mu_{i j}^{t}\right|<\varepsilon$ for all $i$ and $j$. Here $\varepsilon$ is a small value ( $\varepsilon$ is a termination criterion between 0 and 1 ) and $t$ denotes the iteration. Although, the FCM algorithm is efficient, it is very sensitive to the selection of initial values and requires long convergence time in the case of large datasets. To overcome these drawbacks, many clustering algorithms have been introduced recently. Some ${ }^{14,15}$ focus on the objective function optimization and others ${ }^{16,17}$ apply other clustering method to determine initial centers instead of random centers. Also using various kernels in FCM leads to different clustering.

\subsection{I-Fuzzy Partitions}

Intuitionistic fuzzy sets were introduced by Atanassov in $1983^{18}$. It takes into account the membership degree as well as the non-membership degree. In an ordinary fuzzy set, the non-membership degree is the complement of the membership degree, but in intuitionistic fuzzy set the non-membership degree is less than or equal to the complement of the membership degree due to the hesitation degree.
Definition 2. $\quad 19$ An Atanassov intuitionistic fuzzy set (AIFS) $A$ in $X$ is defined by $A=$ $\left\{\left\langle x, \mu_{A}(x), v_{A}(x)\right\rangle \mid x \in X\right\}$ where $\mu_{A}: X \rightarrow[0,1]$ and $v_{A}: X \rightarrow[0,1]$ with $0 \leqslant \mu_{A}(x)+v_{A}(x) \leqslant 1$. For each $x, \mu_{A}(x)$ and $v_{A}(x)$ represent the degree of membership and degree of non-membership of the element $x \in X$ to the AIFS $A$, respectively.

Definition 3. 10 For each IFS $A=$ $\left\{\left\langle x, \mu_{A}(x), v_{A}(x)\right\rangle \mid x \in X\right\}$, the I-fuzzy index for $x \in X$ is defined by $\pi_{A}(x)=1-\mu_{A}(x)-v_{A}(x)$. Ref. 9 generalizes the fuzzy partitions using AIFSs in the following definition. Note that there is an alternative definition of I-fuzzy partition in

Definition 4. Let $X$ be a reference set. Then, a set of AIFSs $A=\left\{A_{1}, \cdots, A_{m}\right\}$ where $A_{i}=\left\langle\mu_{i}, \pi_{i}\right\rangle$ is an I-fuzzy partition if

(i) $\sum_{i=1}^{m} \mu_{i}(x)=1$ for all $x \in X$,

(ii) for all $x \in X$, there is at most one $i$ such that $v_{i}(x)=0$ (there is at most one IFS such that $\mu_{A}(x)+\pi_{A}(x)=1$ for all $\left.x\right)$.

The first condition in the previous definition means that the $\mu_{i}$ are required to define a standard fuzzy partition, i.e., memberships $\mu_{i}$ add to one for all objects $x$. In addition, as $A_{i}$ are required to be an IFS, $\pi_{i}$ stands for the I-fuzzy index for each element $x$ (and each partition element $A_{i}$ ). Therefore, $\mu_{i}(x)+\pi_{i}(x) \leqslant 1$. The second condition constraints the $A_{i}$ so that this inequality is only satisfied as equality for one at most $A_{i}$, i.e., for each x there is only one (or none) $A_{i}$ such that $\mu_{i}(x)+\pi_{i}(x)=1{ }^{10}$.

Proposition 1. ${ }^{10}$ I-fuzzy partitions generalize fuzzy partitions.

\subsection{Hesitant Fuzzy Sets}

The membership degree of a HFS is represented by several possible values in $[0,1]$. The definition is as follows:

Definition 5. ${ }^{4}$ Let $X$ be a fixed set, then a hesitant fuzzy set (HFS) on $X$ in terms of a function $h$ is such that when applied to $X$ returns a subset of $[0,1]$, i.e., $h: X \rightarrow \mathscr{P}([0,1])$.

We use the term typical HFS ${ }^{20}$ when the subsets $h(x)$ are finite. Furthermore, given a set of fuzzy 
sets, a HFS can be defined in accordance with the union of their memberships as follow :

Definition 6. ${ }^{4}$ Let $M=\left\{\mu_{1}, \mu_{2}, \ldots, \mu_{n}\right\}$ be a set of $n$ membership functions and $x \in X$. The HFS associated to $M, h_{M}$ is defined as:

$$
h_{M}(x)=\bigcup_{\mu \in M}\{\mu(x)\} \text {. }
$$

$\mathrm{Xia}$ and $\mathrm{Xu}^{21}$ called $h(x)$ a hesitant fuzzy element (HFE). A hesitant fuzzy element (HFE) is a set of values in $[0,1]$, and a HFS is a set of HFEs, for each $x \in X$.

Definition 7. ${ }^{4}$ Given a HFE, $h$, we define the intuitionistic fuzzy value (IFV) $\operatorname{Aenv}(h)$ as the envelope of $h$, where $\operatorname{Aenv}(h)$ can be represented as $\left(h^{-}, 1-h^{+}\right)$, with $h^{-}=\inf \{\gamma \mid \gamma \in h\}$ and $h^{+}=$ $\sup \{\gamma \mid \gamma \in h\}$. Based on the relationship between the HFEs and IFVs, $\mathrm{Xu}$ and Xia in ${ }^{23}$ defined some new operations on the HFEs. Let $h, h_{1}$ and $h_{2}$ be HFEs and $\lambda$ be a real number then,

- $h^{\lambda}=\cup_{\gamma \in h}\left\{\gamma^{\lambda}\right\}$,

- $\lambda h=\cup_{\gamma \in h}\left\{1-(1-\gamma)^{\lambda}\right\}$

- $h_{1} \oplus h_{2}=\cup_{\gamma_{1} \in h_{1}, \gamma_{2} \in h_{2}}\left\{\gamma_{1}+\gamma_{2}-\gamma_{1} \gamma_{2}\right\}$,

- $h_{1} \otimes h_{2}=\cup_{\gamma_{1} \in h_{1}, \gamma_{2} \in h_{2}}\left\{\gamma_{1} \gamma_{2}\right\}$,

- $h^{c}=\cup_{\gamma \in h}\{1-\gamma\}$,

- $h_{1} \cup h_{2}=\cup_{\gamma_{1} \in h_{1}, \gamma_{2} \in h_{2}} \max \left\{\gamma_{1}, \gamma_{2}\right\}$,

- $h_{1} \cap h_{2}=\cap_{\gamma_{1} \in h_{1}, \gamma_{2} \in h_{2}} \min \left\{\gamma_{1}, \gamma_{2}\right\}$.

Here, we recall some concepts involved in hesitant fuzzy sets which will be used in the present work. They are aggregation operators and correlation coefficients.

\subsubsection{Aggregation Operators}

$\mathrm{Xia}$ and $\mathrm{Xu}$ presented in ${ }^{21}$ some aggregation operators, such as hesitant fuzzy weighted averaging and hesitant fuzzy weighted geometric which are defined as follows. These two operators are a a generalization of the intuitionistic fuzzy weighted averaging (IFWA) operator. Note that there is discussion on the usability of this operator, see Ref. ${ }^{22}$. Nevertheless in our context these two operators seems to be appropriate. Other aggregation operators for HFS can be found in ${ }^{9}$.

Definition 8. ${ }^{21}$ Let $H$ be a hesitant fuzzy set and $h_{i}(i=1, \cdots, n)$ be a collection of HFEs, $h_{i} \in H$, the hesitant fuzzy weighted averaging (HFWA) operator is a mapping $H^{n} \rightarrow H$ such that

$$
\begin{aligned}
\operatorname{HFWA}\left(h_{1}, \cdots, h_{n}\right) & =\oplus_{i=1}^{n}\left(w_{i} h_{i}\right) \\
& =\bigcup_{\gamma_{1} \in h_{1}, \cdots, \gamma_{n} \in h_{n}}\left\{1-\prod_{i=1}^{n}\left(1-\gamma_{i}\right)^{w_{i}}\right\} .
\end{aligned}
$$

where $w=\left(w_{1}, \cdots, w_{n}\right)^{T}$ is a weighting vector with $w_{i} \in[0,1]$ and $\sum_{i=1}^{n} w_{i}=1$. In case of $w=$ $(1 / n, \cdots, 1 / n)^{T}$, then the (HFWA) operator reduces to the hesitant fuzzy averaging (HFA) operator:

$$
\begin{aligned}
H F A\left(h_{1}, \cdots, h_{n}\right) & =\oplus_{i=1}^{n}\left(\frac{1}{n} h_{i}\right) \\
& =\bigcup_{\gamma_{1} \in h_{1}, \cdots, \gamma_{n} \in h_{n}}\left\{1-\prod_{i=1}^{n}\left(1-\gamma_{i}\right)^{\frac{1}{n}}\right\} .
\end{aligned}
$$

Definition 9. Let $h_{i}(i=1, \cdots, n)$ be a collection of HFEs, $w=\left(w_{1}, \cdots, w_{n}\right)^{T}$ be a weighting vector of them, (i.e., $w_{i} \in[0,1]$ and $\sum_{i=1}^{n} w_{i}=1$ ). A generalized hesitant fuzzy weighted geometric (GHFWG) operator is a mapping $H^{n} \rightarrow H$, and

$$
\begin{aligned}
& \operatorname{GHFWA}\left(h_{1}, \ldots, h_{n}\right)=\left(\oplus_{i=1}^{n}\left(w_{i} h_{i}\right)^{\lambda}\right)^{\frac{1}{\lambda}} \\
& =\bigcup_{\gamma_{1} \in h_{1}, \gamma_{2} \in h_{2}, \ldots, \gamma_{n} \in h_{n}}\left\{1-\left(1-\prod_{i=1}^{n}\left(1-\gamma_{i}^{\lambda}\right)^{w_{i}}\right)^{1 / \lambda}\right\} .
\end{aligned}
$$

\subsubsection{Correlation Coefficient}

We say that we have correlation when we have a (linear) relationship between two variables. It is an important concept in data analysis. Because of this, different correlation coefficients have been defined to different types of information (see ${ }^{9}$ for details on its application to HFSs). Chen et al. ${ }^{24}$ defined the informational energy for HFSs and a related correlation between HFSs. We review them below.

Definition 10. ${ }^{24}$ Let $H$ be a typical HFS (i.e., with a finite number of membership degrees) on 
$X=\left\{x_{1}, \cdots, x_{n}\right\}$, the informational energy of the HFS $H$, is defined as follows,

$$
E_{H F S}(H)=\sum_{i=1}^{n}\left(\frac{1}{l\left(h_{M}\left(x_{i}\right)\right)} \sum_{j=1}^{l_{x_{i}}} h_{M_{\sigma}(j)}^{2}\left(x_{i}\right)\right),
$$

where $h_{M}\left(x_{i}\right)$ is a HFE and $h_{M_{\sigma}(j)}\left(x_{i}\right)$ are the $j t h$ largest values of $h_{M}\left(x_{i}\right)$ and $l\left(h_{M}\left(x_{i}\right)\right)$ is the number of values in $h_{M}\left(x_{i}\right)$.

Definition 11. ${ }^{24}$ Let $H_{1}$ and $H_{2}$ be two typical HFSs on $X=\left\{x_{1}, \cdots, x_{n}\right\}$, the correlation between $H_{1}$ and $\mathrm{H}_{2}$ is defined by,

$$
C_{H F S}\left(H_{1}, H_{2}\right)=\sum_{i=1}^{n}\left(\frac{1}{l_{x_{i}}} \sum_{j=1}^{l_{x_{i}}} h_{M_{\sigma}(j)}^{1}\left(x_{i}\right) h_{M_{\sigma}(j)}^{2}\left(x_{i}\right)\right),
$$

where $l_{x_{i}}=\max \left\{l\left(h_{M}^{1}\left(x_{i}\right)\right), l\left(h_{M}^{2}\left(x_{i}\right)\right)\right\}$ and $h_{M}^{1}\left(x_{i}\right)$, $h_{M}^{2}\left(x_{i}\right)$ are a HFE for $H_{1}, H_{2}$, respectively. Let $H_{1}$, $\mathrm{H}_{2}$ be two HFSs, the correlation satisfies:

- $C_{H F S}\left(H_{1}, H_{1}\right)=E_{H F S}\left(H_{1}\right)$;

- $C_{H F S}\left(H_{1}, H_{2}\right)=C_{H F S}\left(H_{2}, H_{1}\right)$.

By using Defs. (11) and (10) the following correlation coefficient is obtained.

Definition 12. Let $H_{1}, H_{2}$ be two typical HFSs on $X=\left\{x_{1}, \cdots, x_{n}\right\}$ the correlation coefficient between $H_{1}$ and $H_{2}$, is,

$$
\rho_{H F S}\left(H_{1}, H_{2}\right)=\frac{C_{H F S}\left(H_{1}, H_{2}\right)}{\left[C_{H F S}\left(H_{1}, H_{1}\right)\right]^{1 / 2}\left[C_{H F S}\left(H_{2}, H_{2}\right)\right]^{1 / 2}} .
$$

\section{H-fuzzy partition}

In this section we introduce a definition for hesitant fuzzy partitions. This definition is for typical hesitant fuzzy sets as we assume that the value of membership degrees is finite.

Definition 13. Let $X=\left\{x_{1}, \cdots, x_{n}\right\}$ be a reference set. Let $H^{*}$ be a HFS on $X H^{*}=\left\{\left\langle x, \widehat{h_{j}}\right\rangle \mid j=\right.$ $1,2, \cdots, m\}$, where $m$ is the number of clusters and $\widehat{h_{j}}=\left\{\mu_{j}^{k} \mid k=1,2, \cdots, \kappa\right\}$ are hesitant fuzzy elements. That is $\widehat{h_{j}}$ is a finite set such that $\widehat{h_{j}} \subseteq[0,1]$ and $\kappa$ is the number of membership degrees in $\widehat{h_{j}}$ (i. e., the cardinality of $\widehat{h_{j}}$ is $\kappa$. We can use $\kappa_{j}$ for $j=1,2, \cdots, m$. However for the sake of simplicity we use $\kappa_{j}=\kappa$ for all $j$ ). Then $H^{*}$ is a hesitant fuzzy partition (H-fuzzy partition) if

$$
\frac{\sum_{j=1}^{m} \sum_{k=1}^{\kappa} \mu_{j}^{k}(x)}{\kappa m} \leqslant 1 \quad \forall x \in X, \quad 0 \leqslant \mu_{j}^{k}(x) \leqslant 1 .
$$

We also consider a more general case in which the set $\widehat{h_{j}}$ is infinite. This is a generalization of the former definition and we use it later to prove that this definition generalizes the one for I-fuzzy partitions discussed above (see Def. (4)).

Definition 14. Let $X=\left\{x_{1}, \cdots, x_{n}\right\}$ be a reference set. Then a set of HFE $H=\left\{\widehat{h_{1}}, \cdots, \widehat{h_{m}}\right\}$, where $\widehat{h_{j}}$ is an infinite set, is a hesitant fuzzy partition if the following holds (note that the following inequality is a reformulation of Eq. (11)):

$$
\sum_{j=1}^{m} \frac{\int_{0}^{1} y \mu_{j}(x)(y) d y}{m \int_{0}^{1} \mu_{j}(x)(y) d y} \leqslant 1 \quad \forall x \in X,
$$

where $\mu_{j}(x)$ is the characteristic function of the set $\widehat{h_{j}}(x)$. We underline that this definition is not only valid for hesitant fuzzy set but also for type-2 fuzzy sets as $\mu_{j}(x)$ can be a fuzzy set. Note that if $\widehat{h_{j}}(x)$ is a single value then we have type- 1 fuzzy sets, if $\widehat{h}_{j}(x)$ is finite set we have typical hesitant fuzzy sets, and when $\widehat{h_{j}}(x)$ is a fuzzy set we have type-2 fuzzy sets. In this case the membership degree of $x$ to cluster $j$ is given by the membership function $\mu_{j}(x)(y)$ for $y \in[0,1]$ instead of having $\mu_{j}(x)$ a single value.

Proposition 2. H-fuzzy partitions generalize I-fuzzy partitions.

Proof. In order to prove this proposition, we need to see that any I-fuzzy partition is also a H-fuzzy partition. That is, conditions in Def. 4 imply the conditions in Def. 13 (if we consider typical HFS) or Def. 14 (if we consider HFS with infinite membership degrees). Because of that, we have to prove that for all $x \in X$ when we have that Def. (4) holds, it also holds Eq. (11) or (12) (depending on whether we understand the I-fuzzy partition as a discrete or a 
continuous set). Without loss of generality we consider a given $x \in X$, from the I-fuzzy partition (Def. (4)). Then $A$ is the set of I-fuzzy sets for $x$ that satisfies the conditions of the I-fuzzy partition. That is, $A=\left\{A_{1}, \cdots, A_{m}\right\}$ with $A_{i}=\left\langle\mu_{i}, \pi_{i}\right\rangle$ and $x$ satisfies (i) and (ii) in Def . (4). Based on ${ }^{4}$ the envelope of hesitant fuzzy set is an intuitionistic fuzzy set. So, for each $A_{i}=\left\langle\mu_{i}, \pi_{i}\right\rangle$, we have $h_{i}^{+}=1-\left(1-\mu_{i}-\pi_{i}\right)$ and $h_{i}^{-}=\mu_{i}$. We consider two models to embed an IFS into HFS.

(i) We model $A_{i}$ as a finite hesitant fuzzy set $h_{i}=\left\{\mu_{i}, 1-\mu_{i}-\pi_{i}\right\}$. Then we prove that $h_{i}=\left\{\mu_{i}, 1-\mu_{i}-\pi_{i}\right\}$ satisfies Def. (13). Note that in this case $\kappa=2$, and therefore for all $h_{i}$ we have $\mu_{i}+\left(1-\mu_{i}-\pi_{i}\right) \leqslant 1$ so, it is clear that

$$
\frac{\sum_{i=1}^{m} 1-\pi_{i}}{2 m} \leqslant 1
$$

(ii) We model $A_{i}$ in terms of an interval, we have that $A_{i}=\left\langle\mu_{i}, \pi_{i}\right\rangle$ corresponds to the interval $\left[\mu_{i}, 1-\mu_{i}-\pi_{i}\right]$. Therefore, we define $h_{i}=$ $\chi_{\left[\mu_{i}, 1-\mu_{i}-\pi_{i}\right]}$ where $\chi$ is a characteristic function. It is clear that

$$
\frac{\int_{0}^{1} y \chi_{\left[\mu_{i}, 1-\mu_{i}-\pi_{i}\right]}(y) d y}{\int_{0}^{1} \chi_{\left[\mu_{i}, 1-\mu_{i}-\pi_{i}\right]}(y) d y} \leqslant 1,
$$

therefore

$$
\frac{\sum_{i=0}^{m} \int_{0}^{1} y \chi_{\left[\mu_{i}, 1-\mu_{i}-\pi_{i}\right]}(y) d y}{m \int_{0}^{1} \chi_{\left[\mu_{i}, 1-\mu_{i}-\pi_{i}\right]}(y) d y} \leqslant 1 .
$$

\subsection{Construction of H-Fuzzy Partitions}

In this section we study how to construct H-fuzzy partitions. Let us consider $r$ fuzzy clustering algorithms (FCM, IFCM, etc.). Then we consider $K$ different executions, one for each application. For example in FCM, we can consider different initial cluster center selection methods, kernels and values of parameter $m$. The application of $r$ fuzzy clustering algorithms with $K$ different parameters to a data set $X=\left\{x_{1}, \cdots, x_{n}\right\}$ results into $r \times K$ fuzzy partitions. We show below how to build the H-fuzzy partition from a set of fuzzy partitions. We will use the following notation. Let $h_{i j}$ denote the set of membership values obtained by the ith clustering algorithm for $j$ th cluster. That is assuming that we have

$$
h_{i j}=\left\{\mu_{i j}^{k} \mid 0 \leqslant \mu_{i j}^{k} \leqslant 1, \quad k=1, \cdots, K\right\} \quad \forall x \in X,
$$

where $i=1, \cdots, r, j=1, \cdots, m$ and $K$ is the number of membership degrees obtained by each clustering algorithm (e.g. from $k$ different points of view). Note that it is not necessary to set the same values of $K$ for all clustering algorithms. So we can use $K_{i}$ for $i=1,2, \cdots, r$. However for the sake of simplicity we use $K_{i}=K$ for all $i$. We arrange the clustering results of $r$ clustering algorithms as a set of hesitant fuzzy sets:

$$
H_{i}=\left\{h_{i 1}, h_{i 2}, \cdots, h_{i m}\right\} \quad i=1, \cdots, r .
$$

Also, we have a set of cluster centers for each clustering algorithm, as follows:

$V_{i j}=\left\{v_{i j}^{k} \mid \quad k=1, \cdots, K\right\} i=1, \cdots, r ; j=1, \cdots, m ; \forall x \in X$.

where $v_{i j}^{k}$ is the cluster center vector obtained by the ith clustering algorithm for the $j$ th cluster with the $k t h$ parametrization, $i=1, \cdots, r$ and $j=1,2, \cdots, m$. We define a new set of cluster centers for each clustering algorithm as follows:

$$
\begin{aligned}
V^{i} & =\left\{v_{i j}^{*} \mid \quad j=1, \cdots, m\right\}, \quad i=1, \cdots, r ; \\
v_{i j}^{*} & =\frac{\sum_{k=1}^{K} v_{i j}^{k}}{K} .
\end{aligned}
$$

Definition 15. We define the H-fuzzy partition, $H^{*}$, inferred from the sets $H_{i}$ and $V^{i}$ as the one obtained from the application of the next steps.

(i) Cluster alignment. Find a correct alignment between the clusters. To do so, we compute the correlation coefficient between every pair of $h_{i l}, h_{p j}$ for $p, i=1,2, \cdots, r$ and $l, j=$ $1,2, \cdots, m$ using Eq. (10) and applying the following rule:

- If $\rho_{H F S}\left(h_{i L}, h_{p J}\right)=\max \left(\rho_{H F S}\left(h_{i, l}, h_{p j}\right)\right)$ then the Lth cluster of the ith clustering algorithm corresponds to the $J t h$ cluster of the pth clustering algorithm. That is for $p, i_{i \neq p}=1,2, \cdots, r$ and $l, j_{l \neq j}=1,2, \cdots, m$. 
In this way the pairs of clusters of two clustering algorithms are associated.

(ii) Membership determination for each cluster. Use an average operator (e.g. Eqs. (6) or (7)) on the membership values of associated clusters to compute the mean membership degree for the $j t h$ cluster. That is, for each cluster compute:

$\widehat{h_{j}}=\operatorname{HFA}\left(h_{1 j}, \cdots, h_{r j}\right)=\oplus_{i=1}^{r}\left(\frac{1}{r}\right) h_{i j} \quad \forall x \in X$.

(iii) Definition of $\mathbf{H}$-fuzzy partition. Define a hesitant fuzzy set of clusters for each $x \in X, H^{*}$ as follow:

$$
H^{*}=\left\{\left\langle x, \widehat{h_{j}}\right\rangle \mid j=1,2, \cdots, m\right\} .
$$

Note that $H^{*}$ is a H-fuzzy partition. In addition note that we have a set of cluster centers for each cluster as follows:

$$
V_{j}=\left\{v_{i j}^{*} \mid i=1,2, \cdots, r\right\} \quad j=1,2, \cdots, m .
$$

This definition lets the user represent various membership degrees and cluster centers and postpone the decision of which of them is preferable selecting later the validity index that is more suitable for various problem. In the example that follows we use F score, but other validity indices exist and could be used for this purpose, as well.

Proposition 3. The above definition builds a $\mathrm{H}$ fuzzy partition.

Proof. Let $H^{*}=\left\{\left\langle x, \widehat{h_{j}}\right\rangle \mid j=1,2, \cdots, m\right\}$ be a set of hesitant fuzzy sets constructed with the definition above and $\widehat{h_{j}}$ obtained using Eq. (17). In this case the cardinality of $\widehat{h_{j}}$ is at most $K^{r}$ where each of its element is in $[0,1]$. Therefore, it is clear that the value of $\kappa$ in Eq. (11) is $K^{r}$. Thus

$$
\frac{\sum_{i=1}^{m} \sum_{k=1}^{K^{r}} \mu_{i}^{k}(x)}{K^{r} m} \leqslant 1 \quad \forall x \in X, \quad 0 \leqslant \mu_{i}^{k}(x) \leqslant 1 .
$$

We illustrate the construction presented in Def. (15) with an artificial example.

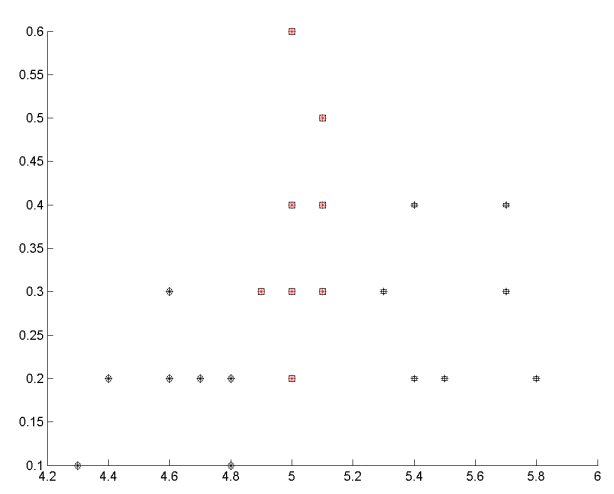

Fig. 1. The Iris 14 data set.

Example 1. We have tested our approach with data from the IRIS data set ${ }^{25}$. This dataset has 150 records and 4 numerical variables. Each record is classified into one of three classes, which are Iris Setosa, Iris Versicolour, and Iris Virginica. To illustrate our method, we have used a subset of 22 records all from class Iris Setosa, and we have only considered two variables (first and fourth variable of the dataset) called it Iris 14 . We have used this small dataset as we can display easily the results graphically. The data is shown in Figure 1.

This dataset has been clustered using two clustering algorithms: FCM (with $\mathrm{m}=1.5$ ) and FCM (with $\mathrm{m}=2.3), r=2$. Each clustering algorithm has been applied with 9 different parameterizations. We consider 3 kernels (cosine distance, Euclidean distance and Mahalanobis distance) and 3 cluster center initialization methods (random, cumulative approach 26 and subtractive clustering ${ }^{27}$ ).

To demonstrate the process of construction of a H-fuzzy partition, we show in Table 1 the obtained results of the point $x=(5,0.3)$ of cluster 2 from the given data set. We select $x=(5,0.3)$ for illustration because this is a point just between two cluster centers. If we select e.g. $x=(4.25,0.1)$, it is easily clustered into correct cluster. We have two hesitant fuzzy sets $H_{1}, H_{2}$ and two sets of cluster centers (one for each cluster). In the following steps we construct the H-fuzzy partition:

- Cluster alignment. To do so, we compute the correlation coefficient between every pair of 
$h_{i l}, h_{p j}$ for $p, i=1,2$ and $l, j=1,2,3$ using Eq. (10). Then the derived correlation matrix is:

$$
\rho=\left(\begin{array}{lll}
1.4848 & 1.4847 & 1.4847 \\
1.4332 & 1.4335 & 1.4334 \\
1.4332 & 1.4335 & 1.4336
\end{array}\right),
$$

based on $\rho$ matrix in this example the pairs $\left(h_{11}, h_{21}\right),\left(h_{12}, h_{22}\right)$ and $\left(h_{13}, h_{23}\right)$ are associated.

\section{- Membership determination for each cluster.} Use the average operator in Eq.(7) on the membership values of associated clusters to compute the mean member ship degree for the $j t h$ cluster. That is, for each cluster $j, j=1,2,3$, compute:

$$
\widehat{h_{j}}=H F A\left(h_{1 j}, h_{2 j}\right)=\left(\frac{1}{2}\right) h_{1 j} \oplus h_{2 j} \quad \forall x \in X .
$$

In this example for considering all information we assume that $\widehat{h_{j}}$ is a multi set. So the number of membership values is $\kappa=81$.

- Definition of H-fuzzy partition. Define a hesitant fuzzy set of clusters for each $x \in X, H^{*}$ as follow:

$$
H^{*}=\left\{\left\langle x, \widehat{h_{j}}\right\rangle \mid j=1,2,3\right\} .
$$

$H^{*}$ is a $\mathrm{H}$-fuzzy partition. Also, we have a set of cluster centers for each cluster as follows:

$$
\begin{aligned}
& V_{1}=\{(4.8037,0.2163),(5.0193,0.2617)\}, \\
& V_{2}=\{(4.9357,0.3235),(5.0086,0.2883)\}, \\
& V_{3}=\{(5.4721,0.299),(5.1838,0.289)\} .
\end{aligned}
$$

In order to evaluate the quality of the final resulting clusters in this H-fuzzy partition, we use the accuracy measure, Fscore. This measurement evaluates the similarity of a clustering to ground truth information of classes ${ }^{28}$. Let $m$ be the number of individual classes. Then, the total Fscore will be computed as the weighted sum of these classes Fscore according to their size. The Fscore can be calculated using Eq. (24), in which $X_{q}$ is a class with the size of $n_{r}$ and $F\left(C_{r}\right)$ is the $F$ score of the class $X_{q}$.

$$
\text { Fscore }=\sum_{r=1}^{m} \frac{n_{r}}{N} F\left(C_{r}\right)
$$

Table 2. The results of first experiments.

\begin{tabular}{lccc}
\hline & \multicolumn{3}{c}{ Fscore of } \\
\cline { 2 - 4 } Dataset & H-fuzzy partition & FCM $(\mathrm{m}=1.5)$ & FCM $(\mathrm{m}=2.3)$ \\
\hline Iris $_{14}$ & 1 & 0.8933 & 0.8933 \\
IRIS & $\mathbf{0 . 9 2 6 4}$ & 0.8933 & 0.8933 \\
\hline
\end{tabular}

For each class $X_{q}, F\left(C_{r}\right)$ finds a cluster $S_{i}$ that agrees with $X_{q}$ better than to the other clusters. $F\left(C_{r}\right)$ is calculated using equation (25), where $P_{S_{i}}$ is the precision (the number of objects in the cluster $S_{i}$ belonging to the class $X_{q}$, divided by the number of objects in the cluster $S_{i}$ ) and $R_{S_{i}}$ is the recall (the number of objects in the cluster $S_{i}$ belonging to the class $X_{q}$, divided by number of objects in the class $\left.X_{q}\right)$.

$$
F\left(C_{r}\right)=\max _{S_{i}}\left\{\frac{2 P_{s_{i}} R_{S_{i}}}{P_{s_{i}}+R_{S_{i}}}\right\}
$$

The obtained Fscore of two executions of FCM (with $m=1.5$ and $m=2.3$ ) and H-fuzzy partition on the two dimensional data set and IRIS data set are illustrated in Table (2).

Table 1. The clustering values for $x=(5,0.3)$.

\begin{tabular}{ccc}
\hline & $x=(5,0.3)$ & \\
\hline & $h_{11}=\{0.9641,0.9640,0.0251,0.0250,0.0250,0.0248,0.0131,0.0130,0.0130\}$ & $v_{11}^{*}=(4.8037,0.2163)$ \\
$H_{1}(5,0.3): \mathrm{FCM}(m=1.5)$ & $h_{12}=\{0.9640,0.9637,0.9636,0.0248,0.0248,0.0130,0.0129,0.0129\}$ & $v_{12}^{*}=(4.9357,0.3235)$ \\
& $h_{13}=\{0.9640,0.9637,0.9636,0.9635,0.0248,0.0248,0.0130,0.0129,0.0129\}$. & $v_{13}^{*}=(5.4721,0.299)$ \\
\hline & $h_{21}=\{0.9614,0.9613,0.9607,0.0256,0.0255,0.0255,0.0135,0.0135,0.0135\}$, & $v_{21}^{*}=(5.0193,0.2617)$ \\
$H_{2}(5,0.3): \mathrm{FCM}(m=2.3)$ & $h_{22}=\{0.96086,0.96083,0.9607,0.0256,0.0251,0.0251,0.0136,0.0134,0.0134\}$, & $v_{22}^{*}=(5.0086,0.2883)$ \\
& $h_{23}=\{0.960863,0.96083,0.96074,0.02565,0.02514,0.01360,0.01342,0.01341\}$. & $v_{23}^{*}=(5.1838,0.289)$ \\
\hline
\end{tabular}




\section{Conclusion and Future Works}

In this paper, we have introduced a definition for H-fuzzy partitions and proposed a method to define them from fuzzy ones. The definition generalizes Ifuzzy partitions and lets the user apply the reliable membership values and cluster centers for new element in various cluster validity indices. In this paper we consider F score to evaluate the obtained H-fuzzy partition.

\section{References}

1. O. Mahamed, E. Andries, S. Ayed, An overview of clustering methods, J. of Intelligent \& Fuzzy Systems 11 (2007), 583-605.

2. X. Chen, A new clustering algorithm based on near neighbor influence, Expert Systems with Applications 42 (2015), 7746-7758.

3. J. Han and M.Kamber, Data Mining:Concepts and Techniques, Morgan Kaufmann Publishers is an imprint of Elsevier (2009), 383-464.

4. V. Torra, Hesitant fuzzy sets, Int. J. of Intelligent Systems 25 (2010), 529-539.

5. V. Torra, Y. Narukawa, On hesitant fuzzy sets and decision, IEEE Int. Conf. on Fuzzy Systems (2009), 1378-1382.

6. B. Zhu, Z.S. Xu, and M.M. Xia. Dual hesitant fuzzy sets, J. of Applied Mathematics (2012), 1-13.

7. G. Qian, H. Wang, and X. Feng, Generalized hesitant fuzzy sets and their application in decision support system, Knowledge-Based Systems 37 (2013), 357365 .

8. R.M. Rodríguez, B. Bedregal, H. Bustince, Y.C. Dong, B. Farhadinia, C. Kahraman, L. Martínez, V. Torra, Y.J. Xu, Z.S. Xu, F. Herrera, A position and perspective analysis of hesitant fuzzy sets on information fusion in decision making. Towards high quality progress, Information Fusion 29 (2016), 89-97.

9. R. M. Rodríguez, L. Martínez, V. Torra, ZS. Xu, and F. Herrera, Hesitant fuzzy sets: state of the art and future directions, Int. J. of Intelligent Systems 29 (2014), 495-524.

10. V. Torra and S. Miyamoto, A definition for I-fuzzy partition, Soft Computing 15 (2011), 363-369.

11. M. Yang and $\mathrm{K}$. Wu, Unsupervised possibilistic clustering, Pattern Recognition 39 (2006), 5-21.

12. R. Coppi, P. Durso, P. Giordani, Fuzzy and possibilistic clustering for fuzzy data, Computational Statistics \& Data Analysis 56 (2012), 915-927.
13. J. C. Bezdek, Pattern Recognition with Fuzzy Objective Function Algorithms, Plenum Press, New York (1981), 191-203.

14. S. J. Nanda, G. Panda, Automatic clustering algorithm based on multi-objective Immunized PSO to classify actions of 3D human models, Engineering Applications of Artificial Intelligence 6 (2013), 1429-1441.

15. K-P. Lin, A novel evolutionary kernel intuitionistic fuzzy C-means clustering algorithm, IEEE Trans. on Fuzzy Systems 22 (5) (2014), 1074-1087.

16. A. Naik, S. C. Satapathy, K. Parvathi, Improvement of initial cluster center of c-means using teaching learning based optimization, Proc.Tech. 6 (2012), 428-435.

17. S. Malek Mohamadi Golsefid, M. H. Fazel Zarandi, Dual-centers type-2 fuzzy clustering framework and its verification and validation indices, Applied Soft Computing, Available online 21 May 2015.

18. K. T. Atanassov, Intuitionistic fuzzy sets, Fuzzy Sets and Systems 20 (1986), 87-96.

19. K. T. Atanassov, On Intuitionistic Fuzzy Sets Theory, Studies in Fuzziness and Soft Computing, Published by Springer (2012), 10-26.

20. B. Bedregal, R. Reiser, H. Bustince, C. Lopez-Molina, V. Torrad, Aggregation functions for typical hesitant fuzzy elements and the action of automorphisms, Information Science 255 (2014), 82-99.

21. M. M. Xia, and Z.S. Xu, Hesitant fuzzy information aggregation in decision making, Int. J. Approximate Reasoning 52 (2011), 395-407.

22. M. Xia, Z. Xu, and H. Liao, Preference relations based on intuitionistic multiplicative information, IEEE Transactions on Fuzzy Systems 21 ( 2013), 113133.

23. Z.S. Xu and M.M. Xia, Distance and similarity measures for hesitant fuzzy sets, Information Sciences 181 (2011), 2128-2138.

24. N. Chen, Z. Xu, M. Xia, Correlation coefcients of hesitant fuzzy sets and their applications to clustering analysis, Applied Mathematical Modelling 37 (2013), 2197-2211.

25. R.A. Fisher, The use of multiple measurements in taxon problems, Annual Eugenics Part II 7 (1936), $179-188$.

26. M. Erisoglu, N. Calis and S. Sakallioglu, A new algorithm for initial cluster centers in k-means algorithm, Pattern Recognition Letters 32 (2011) 1701-1705.

27. SL. Chiu, Fuzzy model identification based on cluster estimatio, J.of Intel and Fuzzy Syst 2 (1994), 267-278.

28. Y. Zhao and G. Karypis, Evaluation of hierarchical clustering algorithms for document datasets, in: Proc. 11 th int. conf on Information and knowledge managment, McLean, Virginia, USA (2002), 515-524. 